\section{THU0219 BENEFICIAL EFFECT OF ANTI-IL-6 BLOCKADE ON INSULIN RESISTANCE AND INSULIN SENSITIVITY IN PATIENTS WITH RHEUMATOID ARTHRITIS}

S. Remuzgo-Martínez ${ }^{1}$, R. López-Mejías ${ }^{1}$, F. Genre ${ }^{1}$, V. Mijares ${ }^{1}$, J. Calvo-Alen ${ }^{2}$,

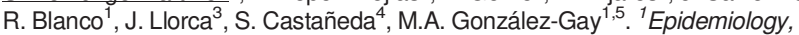
Genetics and Atherosclerosis Research Group on Systemic Inflammatory Diseases, Rheumatology Division, Hospital Universitario Marqués de Valdecilla, IDIVAL, Santander, ${ }^{2}$ Rheumatology Department, Hospital Universitario Araba, Vitoria; ${ }^{3}$ Epidemiology and Computational Biology Department, School of Medicine, University of Cantabria, and CIBER Epidemiología y Salud Pública (CIBERESP), IDIVAL, Santander, ${ }^{4}$ Rheumatology Department, Hospital Universitario La Princesa, IIS-IPrincesa, Madrid; ${ }^{5}$ School of Medicine, University of Cantabria, Santander, Spain

Background: Systemic inflammation, insulin resistance (IR), and endothelial dysfunction have been implicated in the development of cardiovascular disease in rheumatoid arthritis (RA). ${ }^{1-3}$ In this regard, it has been described that the blockade of IL-6, a cytokine involved in the pathogenesis of both RA and atherosclerosis, yields a rapid improvement of endothelial function in RA. ${ }^{4}$ However, there are no studies on the role of anti-IL-6 treatment on IR in patients with RA.

Objectives: To assess whether IL-6 blockade may result in a reduction of insulin serum levels and an improvement of IR in patients with RA.

Methods: 50 Spanish patients on treatment with anti-IL-6 monoclonal antibodyTocilizumab who fulfilled the 2010 classification criteria for $\mathrm{RA}^{5}$ were recruited. Patients with diabetes mellitus or plasma glucose $>110 \mathrm{mg} / \mathrm{dl}$ were excluded. Fasting blood samples were taken for determination of plasma glucose and serum insulin levels immediately prior to (time 0 ) and after (time $60 \mathrm{~min}$ ) Tocilizumab infusion. IR was assessed by the homeostasis model assessment (HOMA) and insulin sensitivity was evaluated by the quantitative insulin sensitivity check index (QUICKI).

Results: A marked reduction in the serum insulin levels was observed following Tocilizumab infusion (mean \pm standard deviation (SD): $10.60 \pm 5.80 \mu \mathrm{U} / \mathrm{ml}$ versus $7.61 \pm 5.08 \mu \mathrm{U} / \mathrm{ml}, p<0.0001)$. In addition, a decrease in the insulin/glucose index was observed in patients with RA after Tocilizumab dose (mean \pm SD: 0.12 \pm 0.06 versus $0.08 \pm 0.05, p<0.0001$ ). Finally, our results disclosed a significant improvement of insulin resistance (HOMA: mean \pm SD: $2.61 \pm 2.05$ versus 1.65 $\pm 1.14, p=0.0003$ ) and insulin sensitivity (QUICKI: mean $\pm S D$ : $0.34 \pm 0.003$ versus $0.37 \pm 0.04, p<0.0001$ ) following Tocilizumab infusion.

Conclusions: Our study confirms a rapid beneficial effect of Tocilizumab on IR and insulin sensitivity in RA patients treated with this drug. It may support the long-term use of drugs that act blocking IL-6 function to reduce the mechanisms implicated in the development of atherosclerosis in RA patients.

\section{REFERENCES:}

[1] Am J Med 2003;114:647-652.

[2] Autoimmun Rev 2016;15:1013-1030.

[3] Autoimmun Rev 2004; 3:301-304.

[4] Atherosclerosis 2011;219:734-736.

[5] Arthritis Rheum 2010;62:2569-2581.

Acknowledgements: This study was supported by European Union FEDER funds and "Fondo de Investigación Sanitaria" (PI15/00525) from "Instituto de Salud Carlos III' (ISCIII, Health Ministry, Spain). It was also partially supported by RETICS Program RD16/0012 (RIER) from ISCIII, and in part by grants from the European IMI BTCure Program. SR-M is supported by funds of the RETICS Program (RIER) RD16/0012/0009 (ISCIII, co-funded by the European Regional Development Fund, ERDF). RL-M is a recipient of a Miguel Servet type I programme fellowship from the ISCIII, co-funded by the European Social Fund (ESF, "Investing in your future") (CP16/00033). FG is a recipient of a Sara Borrell postdoctoral fellowship from the ISCIII, co-funded by ESF (CD15/00095). VM is supported by funds of a Miguel Servet type I programme (CP16/00033) (ISCIII, cofunded by ERDF).

Disclosure of Interest: None declared

DOI: 10.1136/annrheumdis-2018-eular.6998

\section{THU0220 DISCONTINUATION OF BIOLOGIC DMARDS IN A REAL- LIFE POPULATION OF PATIENTS IN REMISSION: OUTCOME AND PREDICTORS}

S. Arnold ${ }^{1}$, V.K. Jaeger ${ }^{1}$, A. Scherer ${ }^{2}$, U.A. Walker ${ }^{1}$, D. Kyburz ${ }^{1} .{ }^{1}$ University Hospital Basel, Basel; ${ }^{2}$ SCQM, Zurich, Switzerland

Background: Data from randomised controlled trials have shown that in patients with sustained remission discontinuation of bDMARD therapy is feasible. However, the criteria for selecting patients that will successfully remain in remission after bDMARD discontinuation remain to be defined.
Objectives: To assess the rate of loss of remission after discontinuation of bDMARDs in a real life setting and to identify predictors in a large cohort of patients.

Methods: Adult RA patients in bDMARD-free remission from the Swiss clinical quality management in rheumatic diseases registry (SCQM) were included. bDMARD-free remission was defined as a discontinuation of the bDMARD and a DAS28 $\leq 2.6$ at a clinical visit within 90 days prior to 30 days after the bDMARD discontinuation. Loss of remission was defined as a DAS28 $>2.6$ or restart of a bDMARD. Patients treated with rituximab were excluded. Kaplan-Meier methods were applied and cox regression was used for multivariable analyses (adjusted for sex, anti-CCP, RF, DAS28, bDMARD Type, CDAl, therapy with conventional DMARDs, number of previous bDMARDs). Missing data were imputed using multiple imputation.

Results: Between 1997 and 2017, 318 patients achieved a bDMARD-free remission (table1). Median observation time was 2.8 years (IQR 1.2-4.9). 241 patients lost remission (76\%), 54\% of those re-started a bDMARD, 34\% experienced a DAS28 flare and $12 \%$ did both. The median time to loss of remission was 0.9 years $(95 \% \mathrm{Cl} 0.7-1.0) .76 \%$ discontinued a TNF $\alpha$-inhibitor, $6 \%$ stopped abatacept, $14 \%$ tocilizumab and $4 \%$ tofacitinib. Women lost remission faster than men (figure $1 \mathrm{~A} ; \mathrm{p}=0.005$ ); this was also seen in multivariable analysis (HR 1.4 $p=0.03$ ). Longer disease duration was associated with a faster loss of remission (figure $1 \mathrm{~B} ; \mathrm{p}=0.03$ ) also in multivariable analysis (HR[4-8 years] 1.6, $\mathrm{p}=0.03$, HR [8-13 years] $1.6, p=0.01, \mathrm{HR}[>13$ years] $1.5, p=0.035$ vs $0-4$ years disease duration). Remission was lost faster by patients who discontinued tofacitinib compared to those who stopped a TNF $\alpha$-inhibitor (HR 2.3, $\mathrm{p}=0.01$ ) also after adjustment (HR 2.0, $p=0.047$ ). There was a trend for a faster loss of remission in patients with a higher disease activity as measured by the CDAl at baseline (figure $1 \mathrm{C} ; \mathrm{p}=0.7$ ); this association was significant in multivariable analysis (HR[CDAI low disease activity] 1.6, $\mathrm{p}=0.03$, HR[CDAI moderate/high disease activity] 2.5, $\mathrm{p}=0.003$, both vs CDAl remission). Patients who received MTX/leflunomide during the observation period lost remission less rapidly (figure $1 \mathrm{D} ; \mathrm{p}=0.006$ ) also in multivariable analysis (HR 0.8, $\mathrm{p}=0.046$ ).

Abstract THU0220 - Table 1. Baseline demographic and disease characteristics of patients with bDMARD-free remission

\begin{tabular}{lcc}
\hline Characteristics & & \% or median (IQR) \\
\hline Male sex & & 26 \\
\hline Age & & $57(46-66)$ \\
Disease duration in years & 0 & $8(4-13)$ \\
Number of previous bDMARDs & $1-2$ & 68 \\
& $\geq 3$ & 27 \\
cDMARD any time during Remission & & 5 \\
Anti-CCP/RF positive & & 64 \\
DAS28 & & $64 / 72$ \\
HAQ & & $2.0(1.5-2.3)$ \\
CDAI & Remission & $0.4(0-1.0)$ \\
& Low Activity & 27 \\
& Moderate-High Activity & 54 \\
\end{tabular}
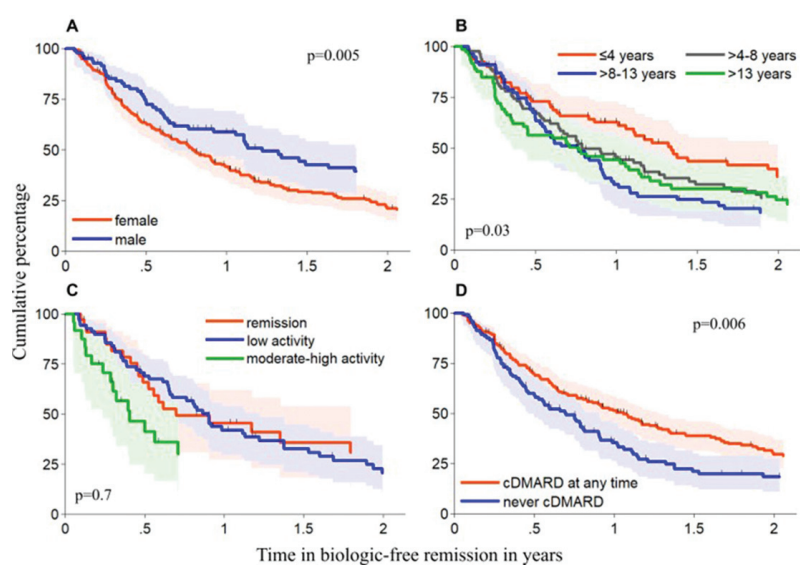

Abstract THU0220 - Figure 1. Kaplan-Meier for time in biologic-free remission according to sex (A), disease duration (B), CDAI (C), conventional DMARD therapy (D)

Conclusions: In a large real-life cohort of RA patients that achieved DAS28 remission and stopped therapy with bDMARD the majority lost remission within less than a year. Predictors for loss of remission included female sex, longer disease duration, a higher CDAl score and absence of cDMARD therapy. This 
suggests that discontinuation of bDMARD should be considered in patients on cDMARD therapy, fulfilling the more stringent remission criteria by CDAI. Disclosure of Interest: None declared

DOI: 10.1136/annrheumdis-2018-eular.3764

\section{THU0221 CLINICAL OUTCOMES OF ABATACEPT VERSUS TNF INHIBITORS IN ACPA-POSITIVE PATIENTS WITH RHEUMATOID ARTHRITIS: DATA FROM THE BIOLOGIC REGISTER KOBIO}

S.-K. Lee ${ }^{1}$, S. Oh ${ }^{2}$, H.-A. Kim ${ }^{3}$, Y.-B. Park ${ }^{4}$, S.-S. Lee ${ }^{5}$, K. Shin ${ }^{1} .{ }^{1}$ Division of Rheumatology, ${ }^{2}$ Department of Biostatistics, Seoul Metropolitan GovernmentSeoul National University Hospital Boramae Medical Center, Seoul; ${ }^{3}$ Division of Rheumatology, Ajou University School of Medicine, Suwon; ${ }^{4}$ Division of Rheumatology, Yonsei University College of Medicine, Seoul; ${ }^{5}$ Division of Rheumatology, Chonnam University Hospital, Gwangju, Korea, Republic of Ireland

Background: Determining the optimal, patient-tailored biologic agent is a new challenge for future guidelines in rheumatoid arthritis (RA) management. Recent studies demonstrated that anti-citrullinated protein antibody (ACPA) -positive patients have better disease activity improvement with abatacept (ABA), yet validation studies are in need.

Objectives: To investigate disease activity changes after treatment with $A B A$ versus TNF inhibitors (TNFi) in Korean ACPA-positive, RA patients.

Methods: Data of RA patients were obtained from the Korean College of Rheumatology Biologics Registry (KOBIO). ACPA-positive patients who were treated with $\mathrm{ABA}$ and TNFi were selected through propensity score matching (1:2, calliper $=0.2 * S D$ ). Clinical outcomes including CDAl changes at the first year of therapy were evaluated, and adjusted drug survival in each group was analysed.

Results: The baseline characteristics of the ABA $(n=97)$ and TNFi $(n=194)$ groups were comparable. The CDAI reduction after 1 year treatment was significant in the ABA group compared with patients who received TNFi ( -16.78 versus $-13.61, p=0.0198$ ) [figure 1]. This was confined when used as the first-line agent $(p=0.0213)$. Proportion of remission and low disease activity in ABA was significant as well $(p=0.041)$. Patients with the lower tertile of ACPA titers treated with $A B A$ had the most significant CDAl reduction compared with the TNFi group $(p=0.0201)$. Drug retention rate (median follow-up 48 months) of ACPA-positive ABA users was also notable compared with TNFi yet did not meet statistical significance (adjusted hazard ratio $0.774, \mathrm{p}=0.086$ )
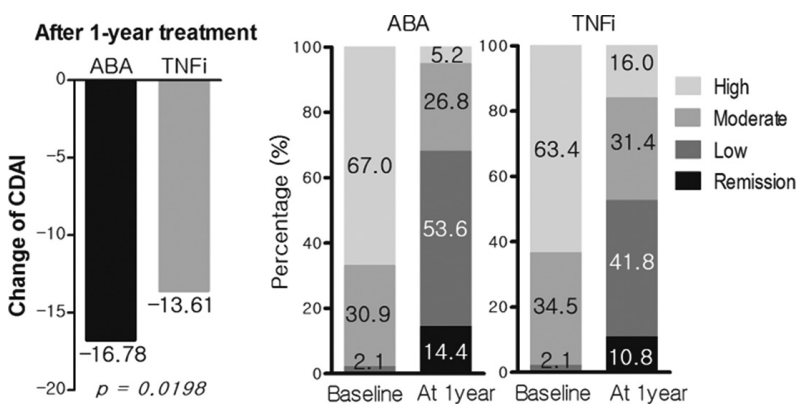

Conclusions: Our data further support that ACPA could also be an important marker to help determine first-line biologic agent of choice among the armamentarium of biologic therapy for RA patients.

Acknowledgements: We would like to thank Song Wha Chae and Evo Alemao for their input and support.

Disclosure of Interest: None declared

DOI: 10.1136/annrheumdis-2018-eular.4357

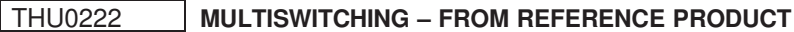 ETANERCEPT TO BIOSIMILAR AND BACK AGAIN - REAL-WORLD DATA FROM A CLINIC-WIDE MULTISWITCH EXPERIENCE}

V. Sigurdardottir, A. Svärd. Center for Clinical Research Dalarna, Falun, Sweden

Background: The etanercept (ETN) biosimilar SB4 was introduced in Sweden in 2016 at a lower price than the reference product etanercept (RPE). Now the reverse is true, as the price of RPE dropped. Switching ETN-treated patients to the lowest priced ETN available is economically favourable. The safety of multiple switches between RPE and biosimilar has been adressed in a psoriasis trial(, ${ }^{1}$ finding no indications of harm. No reports on outcomes of multiswitching in a rheumatology setting are available.
Objectives: To describe the impact of a clinic-wide switch from RPE to SB4 and then back to RPE on disease activity and drug-survival, relating outcomes to a historical cohort of RPE-treated patients.

Methods: Observational study of 145 patients switched from RPE to SB4 (day $0=20 / 4 / 2016$ ) and back to RPE (day $544=16 / 10 / 2017$ ) for economical reasons. Letters were mailed to patients on day 0 and 544, informing them that prescriptions had been changed from RPE to SB4 and from SB4 to $\mathrm{RPE}$ respectively. During days $1-543$, clinicians were allowed to switch patients back to RPE if requested by the patient or if medically indicated. Disease activity data was entered into the Swedish Rheumatology Quality Register (SRQ) at visits. The SRQ was searched retrospectively for data from day -365 to 771 (May 31, 2018). Visits were categorised into visits occurring a) on days -365 to day 0 , b) on days 1-543 and c) on days 544771. A reference cohort of all RPE-treated patients on April 20, 2013 at the clinic was used for comparison.

Results: Numbers and proportions of patients discontinuing SB4 during days $1-543$ in the switching cohort and RPE in the historical cohort are shown in the table 1. On day 544, the 97 patients still treated with SB4 were switched back to RPE. In the switching cohort, DAS28 and CRP did not change significantly when comparing data from visits occurring before the switch from RPE to SB4 to data from visits that occurred on days 1-543 and days 544-636 (fig ure 1). Data from 5 months of additional follow-up, up until day 771 will be presented at the congress. Among the 24 patients that discontinued SB4 during days 1-543 and went back to RPE, no worsening of disease activity parameters was seen during SB4 treatment.

\begin{tabular}{lccc}
\hline & $\begin{array}{c}\text { Switching cohort, } \\
\mathbf{n}=\mathbf{1 4 5}\end{array}$ & $\begin{array}{c}\text { Historical RPE-treated } \\
\text { cohort, } \mathbf{n = 9 8}\end{array}$ & $\mathbf{p}$ \\
\hline $\begin{array}{l}\text { Disontinued SB4/RPE, } \\
\text { total }\end{array}$ & $48(33)$ & $15(15)$ & 0.002 \\
\$Back to RPE & $24(17)$ & Not applicable & N/A \\
$\begin{array}{l}\text { No biologic } \\
\text { \$Non ETN biologic }\end{array}$ & $14(10)$ & $6(6)$ & 0.32 \\
$\begin{array}{l}\text { On ETN (SB4 or RPE) at } \\
\text { day 544 }\end{array}$ & $10(7)$ & $9(9)$ & 0.52 \\
& $121(83)$ & $83(85)$ & 0.80 \\
\end{tabular}

*During days $1-543$ \$ Treatment after discontinuation of SB4/RPE

DAS28, means $\pm 95 \% \mathrm{Cl}$

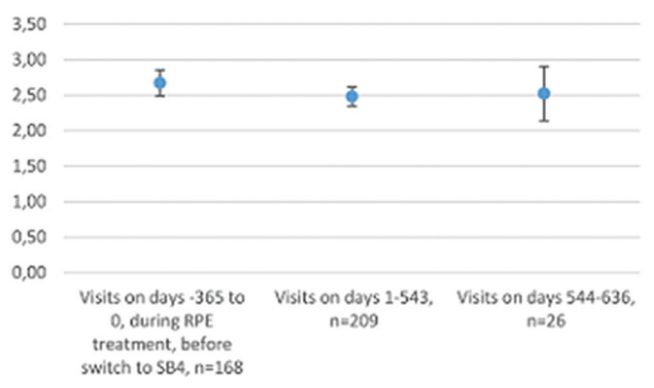

hs-CRP mg/L, means $\pm 95 \% \mathrm{Cl}$

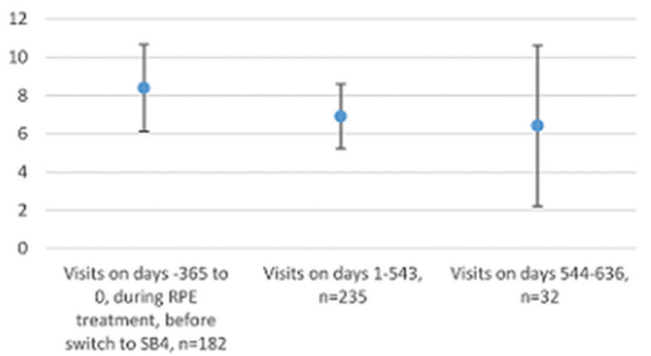

Abstract THU0222 - Figure 1. Mean DAS28 and CRP with $95 \% \mathrm{Cl}$ for 145 patients in the switching cohort according to timepoint of visit..n:s refer to number of visits contributing to data during each period.

Conclusions: The strategy of multiswitching ETN-treated patients has thus far not impacted disease activity negatively in our population. An additional 5 months of follow-up data after the second switch will be presented at the congress. A high proportion of patients discontinued SB4 after the initial switch, as no worsening in disease activity measures was seen in the data we believe this to have been due to nocebo effects. 